\title{
Frecuencia de hábitos orales relacionados con la maloclusión en pacientes de 4 a 12 años: estudio comparativo entre San Luis Potosí- México y Medellín-Colombia, 2016
}

\author{
Frequency of Oral Habits Related to Malocclusion \\ in Patients Aged 4-12: A Comparative Study between \\ San Luis Potosí-Mexico and Medellín-Colombia, 2016
}

Alejandra Arias, Esp. ${ }^{1}$, Gabriel Espinal-Botero $₫$, Esp. ${ }^{2}$, Margarita Ponce, MsC. ${ }^{3}$, Adriana Posada, MsC. ${ }^{4}$, Jorge Nava, CMsC. ${ }^{5}$, Bibiana Salcedo, Esp. ${ }^{6}$

\footnotetext{
1 Universidad Autónoma de San Luis Potosí, México

2 Universidad de Antioquia, Medellín, Colombia

${ }^{3}$ Universidad Autónoma de San Luis Potosí, México

${ }^{4}$ Universidad de Antioquia, Medellín, Colombia

${ }^{5}$ Universidad Autónoma de San Luis Potosí, México

${ }^{6}$ Universidad de Antioquia, Medellín, Colombia
}

Universidad de Antioquia, Medellín, Calle 64 n. ${ }^{0}$ 52-59. Correo electrónico: gabriel.espinal@udea.edu.co

Recibido: 27 de abril del 2017 Aprobado: 29 de mayo del 2017

Cómo citar este artículo: Arias A, Espinal-Botero G, Ponce M, Posada A, Nava J, Salcedo B. Frecuencia de hábitos orales relacionados con la maloclusión en pacientes de 4 a 12 años: estudio comparativo entre San Luis Potosí-México y MedellínColombia, 2016. Rev Nac Odontol. 2018;13(26):1-16. doi: http://dx.doi.org/10.16925/od.v13i26.1814

\section{Resumen}

Introducción: los hábitos orales interfieren en el desarrollo de las funciones del sistema estomatognático, por lo cual son un factor etiológico en el desarrollo de las maloclusiones.

Objetivo: comparar la frecuencia de hábitos orales y su relación con maloclusión en niños de 4 a 12 años de edad en San Luis Potosí-México y Medellín-Colombia.

Método: estudio descriptivo-transversal; la muestra fue por conveniencia de 598 niños de 4 a 12 años de edad (299 niños por ciudad). Para el análisis de datos, se elaboró una tabla de contingencia con chi cuadrado utilizando el programa sPss versión 21.

Resultados: el hábito oral de mayor frecuencia para el total de la muestra fue la onicofagia. Al comparar la muestra en ambas ciudades, en San Luis Potosí la onicofagia presentó la mayor frecuencia y en Medellín, la respiración bucal. En San Luis Potosí, la mayoría de los niños con algún hábito tenían relación molar clase I, excepto aquellos con deglución atípica, quienes presentaron maloclusión clase II. Desde el punto de vista estadístico, la deglución atípica fue el hábito que presentó mayor significancia en relación con la presencia de maloclusiones en el total de la muestra. También se encontró una relación directa entre la presencia de mordida cruzada posterior y la succión digital en San Luis Potosí $(p \leq 0,05)$.

Conclusiones: la deglución atípica y la mordida abierta están directamente relacionadas, lo cual sugiere que la función deglutiva puede ser un factor causal de maloclusión; igualmente, se encontró una relación directa entre succión digital y mordida cruzada posterior.

Palabras clave: hábito, maloclusión, mordida abierta, onicofagia, succión. 


\title{
Frequency of Oral Habits Related to Malocclusion in Patients Aged 4-12: A Comparative Study between San Luis Potosí-Mexico and Medellín-Colombia, 2016
}

\begin{abstract}
Introduction: Oral habits interfere in the development of the functions of the stomatognathic system; therefore, they are an etiological factor in the development of malocclusions.

Aim: To compare the frequency of oral habits and its relationship with malocclusion in children aged 4-12 in San Luis Potosí-Mexico and Medellín-Colombia.

Materials and methods: Cross-sectional, descriptive study. Convenience sample of 598 children from 4 to 12 years old (299 children per city). For data analysis, a contingency table with chi-square was prepared using SPSS v. 21.

Results: The most frequent oral habit for the total sample was onychophagy. When comparing the sample in both cities, onychophagy was highly frequent in San Luis Potosí, while mouth breathing was more common in Medellín. In San Luis Potosí, most children with some habit had a class I molar ratio, except those with atypical swallowing, who exhibited class III malocclusion. From the statistical point of view, atypical swallowing was the habit with the highest significance in relation to the presence of malocclusions in the total sample. A direct relationship between the presence of posterior cross bite and digit sucking in San Luis Potosí ( $p \leq 0.05$ ) was also found.

Conclusions: Atypical swallowing and open bite are directly related, which suggests that the swallowing function can be a causal factor of malocclusion; moreover, a direct relationship between digit sucking and posterior cross bite was found.
\end{abstract}

Keywords: habit, malocclusion, open bite, onychophagy, sucking.

\section{Frequência de hábitos orais relacionados com a maloclusão em pacientes de 4 a 12 anos: estudo comparativo entre San Luis Potosí-México e Medellín-Colômbia, 2016}

\begin{abstract}
Resumo
Introdução: os hábitos orais interferem no desenvolvimento das funções do sistema estomatognático, por isso são um fator etiológico no desenvolvimento das maloclusões.

Objetivo: comparar a frequência de hábitos orais e sua relação com maloclusão em crianças de 4 a 12 anos de idade em Luis Potosí-México e Medellín-Colômbia.

Método: estudo descritivo-transversal; a amostra foi por conveniência de 598 crianças de 4 a 12 anos de idade (299 por cidade). Para a análise dos dados, foi elaborada uma tabela de contingência com qui-quadrado e utilizado o programa sPSs versão 21.

Resultados: 0 hábito oral de maior frequência para o total da amostra foi a onicofagia. Ao comparar a amostra em ambas as cidades, em San Luis Potosí, a onicofagia apresentou a maior frequência e, em Medellín, a respiração bucal. Em San Luis Potosí, a maioria das crianças com algum hábito tinha relação molar classe I, exceto aqueles com deglutição atípica, os quais apresentaram maloclusão classe II. Do ponto de vista estatístico, a deglutição atípica foi o hábito que apresentou maior significância em relação com a presença de maloclusões no total da amostra. Também se constatou uma relação direta entre a presença entre a presença de mordida cruzada posterior e a sucção digital em San Luis Potosí $(p \leq 0,05)$.

Conclusões: a deglutição atípica e a mordida aberta estão diretamente relacionadas, o que sugere que a função da deglutição pode ser um fator causal de maloclusão; além disso, encontrou-se uma relação direta entre sucção digital e mordida cruzada posterior.
\end{abstract}

Palavras-chave: hábito, maloclusão, mordida aberta, onicofagia, sucção. 


\section{Introducción}

Según Clark [1], la oclusión puede ser definida como los contactos entre los dientes superiores e inferiores en todas las posiciones y movimientos mandibulares. Una oclusión funcional es un estado en el cual las superficies oclusales no presentan obstáculos o interferencias para los movimientos mandibulares, y en el que existe la máxima interdigitación cuspídea en oclusión céntrica, respetando todas las reglas de fisiología, anatomía y neurofisiología humana $[2,3]$. Cuando algunas de las características mencionadas están alteradas, se puede llegar a presentar una maloclusión.

La maloclusión dental es una desviación de los dientes de su oclusión ideal, la cual varía entre las personas de acuerdo con diferentes factores ambientales y genéticos. Por otro lado, las maloclusiones esqueléticas se manifiestan como una alteración de forma, tamaño y posición de ambos o alguno de los maxilares [4].

La maloclusión es una anomalía en el desarrollo de los arcos dentarios que ocasiona problemas estéticos y funcionales; las causas más frecuentes son el desarrollo ontogénico, la herencia y condiciones funcionales adquiridas. En las condiciones funcionales adquiridas, la dieta blanda, la respiración bucal y los hábitos orales nocivos están entre las principales causas [5]; la maloclusión se clasifica según Angle en: clase I, clase II división 1 y 2, y clase in según la relación de la cúspide mesiovestibular del primer molar superior con el surco vestibular del primer molar inferior $[1$, p. 78].

Cuando se va a clasificar la maloclusión, se deben tener en cuenta los componentes vertical, sagital y transversal, con lo que se clasifica la maloclusión en mordida abierta [6], mordida profunda $[1$, p. $76,4,7]$, mordida borde-borde anterior, mordida cruzada anterior y mordida cruzada posterior.

Según Leme [8], los hábitos orales son definidos como patrones aprendidos de contracción muscular, los cuales con la práctica se vuelven inconscientes y con frecuencia se incorporan a la personalidad. Se incluyen los hábitos de succión (de pulgar, chupete o biberón), onicofagia, deglución atípica y morderse los labios, entre otros. Los hábitos orales pueden interferir en las funciones del sistema estomatognático y por lo general están relacionados como un factor etiológico de las maloclusiones.

En el artículo de Leme [8, p. 313], algunos autores reportan que $35-50 \%$ de las maloclusiones son causadas por factores externos, y el factor funcional más frecuente que influye en la oclusión son los hábitos orales. Los efectos negativos de los hábitos orales dependerán de su frecuencia, intensidad y duración, así como del tipo de hábito y los patrones de crecimiento hereditarios individuales.

Dos teorías de comportamiento se han utilizado para explicar la etiología de los hábitos orales, en especial de los hábitos de succión no nutritivos: la psicoanalítica, propuesta por Sigmund Freud, y la teoría del aprendizaje. Ambas teorías creen que algunas condiciones con desarrollo normal promueven el origen de los hábitos de succión no nutritivos. De acuerdo con la teoría psicoanalítica, los hábitos de succión son una estimulación placentera de los labios y la boca. La teoría del aprendizaje establece que los hábitos de succión son respuestas adaptativas. Se espera que a los tres años los niños hayan abandonado los hábitos orales. Para los niños que continúan con presencia de hábitos orales después de esta edad, la teoría psicoanalítica sugiere que es un indicativo de trastorno psicológico, causado por la incapacidad de hacer frente a la tensión de la vida. Sin embargo, la teoría del aprendizaje cree que es solo un hábito aprendido. De acuerdo con la teoría psicoanalítica, algunos de los factores etiológicos de hábitos orales son: conflictos familiares, celos, presión de la escuela, ansiedad y estrés [8, p. 313,1, p. 74].

El objetivo de este estudio fue comparar la frecuencia de hábitos orales y su relación con la maloclusión en niños de 4 a 12 años de edad entre las ciudades de San Luis Potosí y Medellín en el 2016.

\section{Materiales y métodos}

Se realizó un estudio descriptivo transversal comparando dos poblaciones diferentes, en el cual se revisaron niños de 4 a 12 años de edad.

La muestra fue determinada por conveniencia, siendo de 299 niños en cada ciudad para un total de 598 niños. Los niños en San Luis Potosí pertenecían a escuelas de nivel preescolar, primaria y secundaria públicas. En Medellín, los niños eran participantes del programa de ortodoncia preventiva e interceptiva del presupuesto participativo de la Secretaría de Salud de la Alcaldía de Medellín, en custodia del Centro de Crecimiento y Desarrollo de la Facultad de Odontología de la Universidad de Antioquia; también había pacientes del programa 
Buen Comienzo de la Alcaldía de Medellín y pacientes con historias clínicas de la Facultad de Odontología de la Universidad de Antioquia. La muestra fue seleccionada de acuerdo con un rango de edad de 4 a 12 años.

Los datos recolectados en ambas ciudades se registraron en un formato de historia clínica utilizado por la Universidad de San Luis Potosí. Las variables estudiadas en cada niño fueron:

\section{- Edad}

- Sexo

- Hábitos orales

- Succión de dedo

- Succión labial

- Biberón

- Chupón

- Bruxismo

- Onicofagia

- Deglución atípica

- Respiración bucal

- Introducción de objetos a la boca

- Relación molar permanente

- Mordida abierta

- Sobremordida vertical

- Sobremordida horizontal

- Mordida borde a borde

- Mordida cruzada anterior

- Mordida cruzada posterior

- Relación molar decidua

Para el análisis, se obtuvieron frecuencias y porcentajes. Además, se realizó la prueba de chi cuadrado con el fin de explorar la relación entre la presencia de hábitos y las maloclusiones, y se asumió una relación estadísticamente significativa en aquella en la que el valor de "p" fuera menor que 0,05 . Para el procesamiento y análisis de los datos, se utilizaron los softwares Microsoft Office Excel y sPss versión 21 .

\section{Resultados}

De los 598 pacientes evaluados, el 52,2\% son hombres, el promedio de edad fue de 7,97 años y la desviación estándar fue de 2,55 años. En cuanto a los hábitos presentes, se observó que el hábito de mayor prevalencia fue la onicofagia en $26,1 \%$ de los niños, seguido de la respiración bucal en 18,6\%. Con valores similares, encontramos que presentaban bruxismo (11,0\%), succión digital (10,7\%), succión labial (10,2\%), deglución atípica (10,4\%) e introducían objetos en su boca (10,7\%). Por último, solo $3,2 \%$ usaban el biberón y 1,0\% usaban chupón.

Del total de la población, al momento de la evaluación, 419 niños presentaban molares permanentes, de los cuales $64,4 \%$ tenían relación clase I; $19,8 \%$, clase II; y $15,8 \%$, clase III, según la clasificación de Angle. Los 177 restantes estaban en dentición decidua: $45,8 \%$ presentaban plano terminal recto; $29,4 \%$, escalón mesial; $16,4 \%$, mesial exagerado; y $8,5 \%$, escalón distal.

En el análisis del plano vertical, se encontró presencia de mordida abierta anterior en el 9,4\% y mordida profunda en el 20,9\%; en el plano sagital, se encontró sobremordida horizontal en el 14,2\% de los niños, mordida borde a borde en el $8,4 \%$ y mordida cruzada anterior en el 7,4\%; en cuanto al plano transversal, se observó mordida cruzada posterior en el 6,4\% de los pacientes.

En el análisis bivariado, se encontró que los pacientes procedentes de San Luis Potosí con edades entre 10 y 12 años presentaron con mayor frecuencia el hábito de introducción de objetos en la boca $(60,7 \%)$, onicofagia $(49,0 \%)$ y succión labial (46,7\%); mientras que el hábito de biberón tuvo mayor frecuencia en quienes estaban entre 4 y 6 años de edad. Al comparar los resultados anteriores con los pacientes de Medellín, se observó similitud en la presencia de los hábitos de onicofagia $(44,6 \%)$ y respiración bucal $(49,2 \%)$ en los niños con edades entre 10 y 12 años; y en los niños de 4 a 6 años en los hábitos de uso de biberón (85,7\%); en todos los anteriores se encontraron diferencias estadísticamente significativas ( $\mathrm{p}<0,005$; Tabla 1$)$.

En Medellín, la presencia de los hábitos de succión labial $(62,5 \%)$ e introducción de objetos en la boca $(100,0 \%)$ se dio en los niños entre 4 y 6 años de edad.

Al comparar los hábitos según el sexo por ciudad, se encontró que en San Luis Potosí son las mujeres las que muestran mayor presencia de hábitos en comparación con los hombres; solo la succión digital se dio en igual proporción y el hábito de deglución atípica fue un tanto mayor en los hombres (55,2\%). En los pacientes de Medellín, las mujeres presentaron con mayor frecuencia los hábitos de introducción de objetos en la boca y el uso de chupón $(66,7 \%)$, deglución atípica $(57,6 \%)$, succión digital $(53,6 \%)$ y respiración bucal $(50,8 \%)$; el resto de los hábitos estudiados se encontraban con mayor frecuencia en los hombres. Las diferencias 
Tabla 1. Frecuencia de hábitos bucales según edad por ciudad, 2016

\begin{tabular}{|c|c|c|c|c|c|c|c|c|c|c|c|c|c|c|c|c|}
\hline \multirow{3}{*}{$\begin{array}{l}\text { Hábitos } \\
\text { bucales }\end{array}$} & \multirow{3}{*}{$\begin{array}{c}\text { Tamaño } \\
\text { de la } \\
\text { muestra }\end{array}$} & \multicolumn{7}{|c|}{ San Luis Potosí } & & \multicolumn{7}{|c|}{ Medellín } \\
\hline & & \multicolumn{2}{|c|}{4 a 6 años } & \multicolumn{2}{|c|}{7 a 9 años } & \multicolumn{2}{|c|}{10 a 12 años } & \multirow{2}{*}{ P-valor } & \multirow{2}{*}{$\begin{array}{c}\text { Tamaño } \\
\text { de la } \\
\text { muestra }\end{array}$} & \multicolumn{2}{|c|}{4 a 6 años } & \multicolumn{2}{|c|}{7 a 9 años } & \multicolumn{2}{|c|}{10 a 12 años } & \multirow{2}{*}{ P-valor } \\
\hline & & $\mathrm{n}$ & $\%$ & n & $\%$ & $\mathrm{n}$ & $\%$ & & & $\mathrm{n}$ & $\%$ & $\mathrm{n}$ & $\%$ & $\mathrm{n}$ & $\%$ & \\
\hline \multicolumn{17}{|c|}{ Succión digital } \\
\hline Sí & 36 & 16 & 44,4 & 10 & 27,8 & 10 & 27,8 & \multirow{2}{*}{0,303} & 28 & 10 & 35,7 & 9 & 32,1 & 9,0 & 32,1 & \multirow{2}{*}{0,950} \\
\hline No & 263 & 83 & 31,6 & 93 & 35,4 & 87 & 33,1 & & 271 & 89 & 32,8 & 93 & 34,3 & 89,0 & 32,8 & \\
\hline \multicolumn{17}{|c|}{ Succión labial } \\
\hline Sí & 45 & 5 & 11,1 & 19 & 42,2 & 21 & 46,7 & \multirow{2}{*}{0,003} & 16 & 10 & 62,5 & 3 & 18,8 & 3 & 18,8 & \multirow{2}{*}{0,037} \\
\hline No & 254 & 94 & 37,0 & 84 & 33,1 & 76 & 29,9 & & 283 & 89 & 31,4 & 99 & 35,0 & 95 & 33,6 & \\
\hline \multicolumn{17}{|l|}{ Biberón } \\
\hline Sí & 12 & 11 & 91,7 & 1 & 8,3 & 0 & 0,0 & \multirow{2}{*}{0,000} & 7 & 6 & 85,7 & 1 & 14,3 & 0 & 0,0 & \multirow{2}{*}{0,010} \\
\hline No & 287 & 88 & 30,7 & 102 & 35,5 & 97 & 33,8 & & 292 & 93 & 31,8 & 101 & 34,6 & 98 & 33,6 & \\
\hline \multicolumn{17}{|l|}{ Chupón } \\
\hline Sí & 0 & 0 & 0,0 & 0 & 0,0 & 0 & 0,0 & \multirow{2}{*}{-} & 6 & 3 & 50,0 & 3 & 50,0 & 0 & 0,0 & \multirow{2}{*}{0,225} \\
\hline No & 299 & 99 & 33,1 & 103 & 34,4 & 97 & 32,4 & & 293 & 96 & 32,8 & 99 & 33,8 & 98 & 33,4 & \\
\hline \multicolumn{17}{|l|}{ Bruxismo } \\
\hline Sí & 31 & 13 & 41,9 & 8 & 25,8 & 10 & 32,3 & & 35 & 8 & 22,9 & 18 & 51,4 & 9 & 25,7 & \\
\hline No & 268 & 86 & 32,1 & 95 & 35,4 & 87 & 32,5 & , & 264 & 91 & 34,5 & 84 & 31,8 & 89 & 33,7 & , \\
\hline Onicofagi & & & & & & & & & & & & & & & & \\
\hline Sí & 100 & 16 & 16,0 & 35 & 35,0 & 49 & 49,0 & 0000 & 56 & 19 & 33,9 & 12 & 21,4 & 25 & 44,6 & \\
\hline No & 199 & 83 & 41,7 & 68 & 34,2 & 48 & 24,1 & 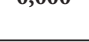 & 243 & 80 & 32,9 & 90 & 37,0 & 73 & 30 & , \\
\hline Deglución & ípica & & & & & & & & & & & & & & & \\
\hline Sí & 29 & 11 & 37,9 & 6 & 20,7 & 12 & 41,4 & & 33 & 13 & 39,4 & 11 & 33,3 & 9 & 27,3 & \\
\hline No & 270 & 88 & 32,6 & 97 & 35,9 & 85 & 31,5 & $0,2+7$ & 266 & 86 & 32,3 & 91 & 34,2 & 89 & 33,5 & 0,070 \\
\hline Respiracic & bucal & & & & & & & & & & & & & & & \\
\hline Sí & 50 & 21 & 42,0 & 20 & 40,0 & 9 & 18 & 0,054 & 61 & 11 & 18,0 & 20 & 32,8 & 30 & 49,2 & 002 \\
\hline No & 249 & 78 & 31,3 & 83 & 33,3 & 88 & 35,3 & 0,054 & 238 & 88 & 37,0 & 82 & 34,5 & 68 & 28,6 & 0,003 \\
\hline Objetos a & boca & & & & & & & & & & & & & & & \\
\hline Sí & 61 & 7 & 11,5 & 17 & 27,9 & 37 & 60,7 & 000 & 3 & 3 & 100 & 0 & 0,0 & 0 & 0,0 & 7 \\
\hline No & 238 & 92 & 38,7 & 86 & 36,1 & 60 & 25,2 & 0,000 & 296 & 96 & 32,4 & 102 & 34,5 & 98 & 33,1 & 0,017 \\
\hline
\end{tabular}

Fuente: elaboración propia

anteriores no fueron estadísticamente significativas (Tabla 2).

En cuanto a la relación entre la presencia de los hábitos y la relación molar permanente según la clasificación de Angle, en San Luis Potosí, se reportó que la mayoría de los niños con los hábitos estudiados tenían relación molar clase i; excepto en la deglución atípica, quienes presentaron relación molar clase III $(47,4 \%)$, siendo esta diferencia estadísticamente significativa $(p=0,000)$. De manera similar, se observan los resultados en Medellín, donde la mayoría de los niños con hábitos bucales presentaron relación molar clase I, excepto en el hábito de biberón, que tuvo relación clase III; dicha diferencia no fue estadísticamente significativa (Tabla 3).

En los pacientes con dentición decidua, en San Luis Potosí las diferencias no son estadísticamente significativas. Se observa que la mayoría de los niños presentan plano terminal recto, excepto para el hábito de succión labial, quienes presentan sobre todo escalón distal $(40,0 \%)$ y para el hábito de onicofagia, escalón mesial (57,1\%). En Medellín, la mayor parte de los pacientes con los hábitos de succión digital, uso de chupón, deglución atípica y respiración bucal mostraron plano terminal recto y el resto registró escalón mesial (Tabla 4). 
Tabla 2. Frecuencia de hábitos bucales según sexo por ciudad, 2016

\begin{tabular}{|c|c|c|c|c|c|c|c|c|c|c|c|c|}
\hline \multirow{3}{*}{$\begin{array}{l}\text { Hábitos } \\
\text { bucales }\end{array}$} & \multirow{3}{*}{$\begin{array}{l}\text { Tamaño de } \\
\text { la muestra }\end{array}$} & \multicolumn{5}{|c|}{ San Luis Potosí } & \multirow{3}{*}{$\begin{array}{l}\text { Tamaño de } \\
\text { la muestra }\end{array}$} & \multicolumn{5}{|c|}{ Medellín } \\
\hline & & \multicolumn{2}{|c|}{ Hombre } & \multicolumn{2}{|c|}{ Mujer } & \multirow{2}{*}{ P-valor } & & \multicolumn{2}{|c|}{ Hombre } & \multicolumn{2}{|c|}{ Mujer } & \multirow{2}{*}{ P-valor } \\
\hline & & $\mathbf{n}$ & $\%$ & $\mathrm{n}$ & $\%$ & & & $\mathbf{n}$ & $\%$ & n & $\%$ & \\
\hline \multicolumn{13}{|c|}{ Succión digital } \\
\hline Sí & 36 & 18 & 50,0 & 18 & 50,0 & \multirow{2}{*}{0,528} & 28 & 13 & 46,4 & 15 & 53,6 & \multirow{2}{*}{0,578} \\
\hline No & 263 & 129 & 49,0 & 134 & 51,0 & & 271 & 126 & 46,5 & 145 & 53,5 & \\
\hline \multicolumn{13}{|c|}{ Succión labial } \\
\hline Sí & 45 & 17 & 37,8 & 28 & 62,2 & \multirow{2}{*}{0,067} & 16 & 9 & 56,3 & 7 & 43,8 & \multirow{2}{*}{0,292} \\
\hline No & 254 & 130 & 51,2 & 124 & 48,8 & & 283 & 130 & 45,9 & 153 & 54,1 & \\
\hline \multicolumn{13}{|l|}{ Biberón } \\
\hline Sí & 12 & 3 & 25,0 & 9 & 75,0 & \multirow{2}{*}{0,077} & 7 & 4 & 57,1 & 3 & 42,9 & \multirow{2}{*}{0,423} \\
\hline No & 287 & 144 & 50,2 & 143 & 49,8 & & 292 & 135 & 46,2 & 157 & 53,8 & \\
\hline \multicolumn{13}{|l|}{ Chupón } \\
\hline Sí & 0 & 0 & 0,0 & 0 & 0,0 & & 6 & 2 & 33,3 & 4 & 66,7 & \multirow{2}{*}{0,411} \\
\hline No & 299 & 147 & 49,2 & 152 & 50,8 & & 293 & 137 & 46,8 & 156 & 53,2 & \\
\hline \multicolumn{13}{|c|}{ Bruxismo } \\
\hline Sí & 31 & 12 & 38,7 & 19 & 61,3 & \multirow{2}{*}{0,149} & 35 & 19 & 54,3 & 16 & 45,7 & \multirow{2}{*}{0,211} \\
\hline No & 268 & 135 & 50,4 & 133 & 49,6 & & 264 & 120 & 45,5 & 144 & 54,5 & \\
\hline \multicolumn{13}{|c|}{ Onicofagia } \\
\hline Sí & 100 & 49 & 49,0 & 51 & 51,0 & \multirow{2}{*}{0,533} & 56 & 29 & 51,8 & 27 & 48,2 & 0232 \\
\hline No & 199 & 98 & 49,2 & 101 & 50,8 & & 243 & 110 & 54,3 & 133 & 54,7 & 0,252 \\
\hline Deglució & atípica & & & & & & & & & & & \\
\hline Sí & 29 & 16 & 55,2 & 13 & 44,8 & 021 & 33 & 14 & 42,4 & 19 & 57,6 & م \\
\hline No & 270 & 131 & 48,5 & 139 & 51,5 & 0,314 & 266 & 125 & 47,0 & 141 & 53 & $0,3 / 9$ \\
\hline Respirac & n bucal & & & & & & & & & & & \\
\hline Sí & 50 & 22 & 44,0 & 28 & 56,0 & & 61 & 30 & 49,2 & 31 & 50,8 & \\
\hline No & 249 & 125 & 50,2 & 124 & 49,8 & 0,200 & 238 & 109 & 45,8 & 129 & 54,2 & 0,371 \\
\hline Objetos & la boca & & & & & & & & & & & \\
\hline Sí & 61 & 30 & 49,2 & 31 & 50,8 & 0556 & 3 & 1 & 33,3 & 2 & 66,7 & 0553 \\
\hline No & 238 & 117 & 49,2 & 121 & 50,8 & 0,556 & 296 & 138 & 46,6 & 158 & 53,4 & 0,553 \\
\hline
\end{tabular}

Fuente: elaboración propia

Tabla 3. Frecuencia de hábitos bucales según la relación oclusal en dientes permanentes por ciudad, 2016

\begin{tabular}{|c|c|c|c|c|c|c|c|c|c|c|c|c|c|c|c|c|}
\hline \multirow{3}{*}{$\begin{array}{l}\text { Hábitos } \\
\text { bucales }\end{array}$} & \multirow{3}{*}{$\begin{array}{c}\text { Tamaño } \\
\text { de la } \\
\text { muestra }\end{array}$} & \multicolumn{7}{|c|}{ San Luis Potosí } & \multicolumn{8}{|c|}{ Medellín } \\
\hline & & \multicolumn{2}{|c|}{ Clase I } & \multicolumn{2}{|c|}{ Clase II } & \multicolumn{2}{|c|}{ Clase III } & \multirow{2}{*}{ P-valor } & \multirow{2}{*}{$\begin{array}{c}\text { Tamaño } \\
\text { de la } \\
\text { muestra }\end{array}$} & \multicolumn{2}{|c|}{ Clase I } & \multicolumn{2}{|c|}{ Clase II } & \multicolumn{2}{|c|}{ Clase III } & \multirow{2}{*}{ P-valor } \\
\hline & & $\mathrm{n}$ & $\%$ & $\mathrm{n}$ & $\%$ & $\mathrm{n}$ & $\%$ & & & $\mathrm{n}$ & $\%$ & $\mathrm{n}$ & $\%$ & $\mathrm{n}$ & $\%$ & \\
\hline \multicolumn{17}{|c|}{ Succión digital } \\
\hline Sí & 21 & 15 & 71,4 & 4 & 19,0 & 2 & 9,5 & 0,862 & 17 & 9 & 52,9 & 6 & 35,3 & 2 & 11,8 & 0,059 \\
\hline No & 186 & 122 & 65,6 & 44 & 23,7 & 20 & 10,8 & & 195 & 126 & 64,6 & 27 & 13,6 & 42 & 21,5 & \\
\hline \multicolumn{17}{|c|}{ Succión labial } \\
\hline Sí & 40 & 23 & 57,5 & 14 & 35,0 & 3 & 7,5 & 0,135 & 7 & 5 & 71,4 & 0 & 0,0 & 2 & 28,6 & 0,495 \\
\hline No & 167 & 114 & 68,3 & 34 & 20,4 & 19 & 11,4 & & 205 & 130 & 63,4 & 33 & 16,1 & 42 & 20,5 & \\
\hline
\end{tabular}


(viene)

\begin{tabular}{|c|c|c|c|c|c|c|c|c|c|c|c|c|c|c|c|c|}
\hline \multirow{3}{*}{$\begin{array}{l}\text { Hábitos } \\
\text { bucales }\end{array}$} & \multirow{3}{*}{$\begin{array}{c}\text { Tamaño } \\
\text { de la } \\
\text { muestra }\end{array}$} & \multicolumn{7}{|c|}{ San Luis Potosí } & \multicolumn{8}{|c|}{ Medellín } \\
\hline & & \multicolumn{2}{|c|}{ Clase I } & \multicolumn{2}{|c|}{ Clase II } & \multicolumn{2}{|c|}{ Clase III } & \multirow{2}{*}{ P-valor } & \multirow{2}{*}{$\begin{array}{c}\text { Tamaño } \\
\text { de la } \\
\text { muestra }\end{array}$} & \multicolumn{2}{|c|}{ Clase I } & \multicolumn{2}{|c|}{ Clase II } & \multicolumn{2}{|c|}{ Clase III } & \multirow{2}{*}{ P-valor } \\
\hline & & $\mathrm{n}$ & $\%$ & $\mathrm{n}$ & $\%$ & $\mathrm{n}$ & $\%$ & & & $\mathrm{n}$ & $\%$ & $\mathrm{n}$ & $\%$ & $\mathrm{n}$ & $\%$ & \\
\hline \multicolumn{17}{|l|}{ Biberón } \\
\hline Sí & 1 & 1 & 100 & 0 & 0,0 & 0 & 0,0 & \multirow{2}{*}{0,774} & 1 & 0 & 0,0 & 0 & 0,0 & 1 & 100 & \multirow{2}{*}{0,147} \\
\hline No & 206 & 136 & 66,0 & 48 & 23,2 & 22 & 10,7 & & 211 & 135 & 64,0 & 33 & 15,6 & 43 & 20,4 & \\
\hline \multicolumn{17}{|l|}{ Chupón } \\
\hline Sí & 0 & 0 & 0 & 0 & 0,0 & 0 & 0,0 & \multirow{2}{*}{-} & 33 & 2 & 66,7 & 1 & 33,3 & 0 & 0,0 & \multirow{2}{*}{0,534} \\
\hline No & 207 & 137 & 66,2 & 48 & 23,2 & 22 & 10,6 & & 209 & 133 & 63,6 & 32 & 15,3 & 44 & 21,1 & \\
\hline \multicolumn{17}{|l|}{ Bruxismo } \\
\hline Sí & 17 & 8 & 47,1 & 5 & 29,4 & 4 & 23,5 & \multirow{2}{*}{0,120} & 25 & 17 & 68,0 & 4 & 16,0 & 4 & 16,0 & \multirow{2}{*}{0,821} \\
\hline No & 190 & 129 & 67,9 & 43 & 22,6 & 18 & 9,5 & & 187 & 118 & 63,1 & 29 & 15,5 & 40 & 21,4 & \\
\hline \multicolumn{17}{|c|}{ Onicofagia } \\
\hline Sí & 92 & 63 & 68,5 & 20 & 21,7 & 9 & 9,8 & \multirow[t]{2}{*}{0,822} & 39 & 24 & 61,5 & 8 & 20,5 & 7 & 17,9 & \multirow[t]{2}{*}{0,617} \\
\hline No & 115 & 74 & 64,3 & 28 & 24,3 & 13 & 11,3 & & 173 & 111 & 64,2 & 25 & 14,5 & 37 & 21,4 & \\
\hline \multicolumn{17}{|c|}{ Deglución atípica } \\
\hline Sí & 19 & 5 & 26,3 & 5 & 26,3 & 9 & 47,4 & \multirow{2}{*}{0,000} & 23 & 10 & 43,5 & 4 & 17,4 & 9 & 39,1 & \multirow{2}{*}{0,052} \\
\hline No & 188 & 132 & 70,2 & 43 & 22,9 & 13 & 11,3 & & 189 & 125 & 66,1 & 29 & 15,3 & 35 & 18,5 & \\
\hline \multicolumn{17}{|c|}{ Respiración bucal } \\
\hline Sí & 29 & 14 & 48,3 & 9 & 31,0 & 6 & 20,7 & 000 & 51 & 32 & 62,7 & 9 & 17,6 & 10 & 19,6 & 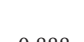 \\
\hline No & 178 & 123 & 96,1 & 39 & 21,9 & 16 & 9,0 & ו וכט, & 161 & 103 & 64,0 & 24 & 14,9 & 34 & 21,1 & 0,000 \\
\hline Objetos a & boca & & & & & & & & & & & & & & & \\
\hline Sí & 51 & 36 & 70,6 & 9 & 17,6 & 6 & 11,8 & 066 & 0 & 0 & 0,0 & 0 & 0,0 & 0 & 0,0 & \\
\hline No & 156 & 101 & 64,7 & 39 & 25,0 & 16 & 10,3 & 0,092 & 212 & 135 & 63,7 & 33 & 15,6 & 44 & 20,8 & - \\
\hline
\end{tabular}

Fuente: elaboración propia

$\mathrm{Al}$ analizar la relación entre la presencia de hábitos y mordida profunda, se encontraron diferencias estadísticamente significativas $(\mathrm{p} \leq 0,05)$ en San Luis Potosí en pacientes con hábitos de succión labial $(13,3 \%)$ y deglución atípica con mordida abierta anterior (17,2\%); de igual forma, se encontró una relación significativa entre la succión labial con mordida profunda (27,8\%). En contraste, en Medellín se observan diferencias estadísticamente significativas en los que presentan succión digital $(64,3 \%)$, bruxismo $(2,9 \%)$ y deglución atípica $(63,6 \%)$ con mordida abierta. Por último, se observaron diferencias entre aquellos que no presentan el hábito de deglución atípica con mordida profunda (22,9\%; Tabla 5).

Al analizar la relación entre la presencia de hábitos y el tipo de sobremordida horizontal, se encontraron diferencias estadísticamente significativas $(\mathrm{p} \leq 0,05)$ en pacientes de San Luis Potosí con hábito de respiración bucal y mordida cruzada anterior (14\%), y en Medellín, en quienes presentan succión digital con sobremordida horizontal (28,6\%; Tabla 6).

$\mathrm{Al}$ analizar la relación del tipo de hábito con la mordida cruzada posterior, en San Luis Potosí, se encontró diferencia estadísticamente significativa $(\mathrm{p} \leq 0,05)$ en aquellos pacientes que presentan succión digital (13,9\%; Tabla 7).

\section{Discusión}

Los hábitos orales deletéreos a menudo se llaman dañinos o parafuncionales e incluyen: succión del pulgar, alimentación con biberón, empuje lingual, onicofagia, mordimiento de los labios y respiración bucal. Estos hábitos tienen una influencia directa en la calidad de vida y pueden afectar el sistema estomatognático [9] (Tabla 8). 
Tabla 4. Frecuencia de hábitos bucales según la relación oclusal en dientes deciduos por ciudad, 2016

\begin{tabular}{|c|c|c|c|c|c|c|c|c|c|c|c|c|c|c|c|c|c|c|c|c|}
\hline \multirow{3}{*}{$\begin{array}{l}\text { Hábitos } \\
\text { bucales }\end{array}$} & \multirow{3}{*}{$\begin{array}{c}\text { Tamaño } \\
\text { de la } \\
\text { muestra }\end{array}$} & \multicolumn{8}{|c|}{ San Luis Potosí } & \multirow{3}{*}{ P-valor } & \multirow{3}{*}{$\begin{array}{l}\text { Tamaño } \\
\text { de la } \\
\text { muestra }\end{array}$} & \multicolumn{8}{|c|}{ Medellín } & \multirow{3}{*}{ p-valor } \\
\hline & & \multicolumn{2}{|c|}{$\begin{array}{c}\text { Plano } \\
\text { vertical }\end{array}$} & \multicolumn{2}{|c|}{$\begin{array}{l}\text { Escalón } \\
\text { mesial }\end{array}$} & \multicolumn{2}{|c|}{$\begin{array}{l}\text { Escalón } \\
\text { distal }\end{array}$} & \multicolumn{2}{|c|}{$\begin{array}{c}\text { Mesial } \\
\text { exagerado }\end{array}$} & & & \multicolumn{2}{|c|}{$\begin{array}{c}\text { Plano } \\
\text { vertical }\end{array}$} & \multicolumn{2}{|c|}{$\begin{array}{c}\text { Escalón } \\
\text { mesial }\end{array}$} & \multicolumn{2}{|c|}{$\begin{array}{l}\text { Escalón } \\
\text { distal }\end{array}$} & \multicolumn{2}{|c|}{$\begin{array}{c}\text { Mesial } \\
\text { exagerado }\end{array}$} & \\
\hline & & $\mathbf{n}$ & $\%$ & $\mathbf{n}$ & $\%$ & $\mathrm{n}$ & $\%$ & $\mathbf{n}$ & $\%$ & & & $\mathbf{n}$ & $\%$ & $\mathrm{n}$ & $\%$ & $\mathbf{n}$ & $\%$ & $\mathbf{n}$ & $\%$ & \\
\hline \multicolumn{21}{|c|}{ Succión digital } \\
\hline Sí & 14 & 10 & 71,4 & 4 & 28,6 & 0 & 0,0 & 0 & 0,0 & \multirow{2}{*}{0,118} & 11 & 7 & 63,6 & 1 & 9,1 & 0 & 0,0 & 3 & 27,3 & \multirow{2}{*}{0,250} \\
\hline No & 76 & 35 & 46,1 & 18 & 23,7 & 14 & 18,4 & 9 & 11,8 & & 76 & 29 & 38,2 & 29 & 38,2 & 1 & 1,3 & 17 & 22,4 & \\
\hline \multicolumn{21}{|c|}{ Succión labial } \\
\hline Sí & 5 & 1 & 20,0 & 1 & 20,0 & 2 & 40,0 & 1 & 20,0 & \multirow{2}{*}{0,313} & 9 & 2 & 22,2 & 3 & 33,3 & 0 & 0,0 & 4 & 44,4 & \multirow{2}{*}{0,389} \\
\hline No & 85 & 44 & 51,8 & 21 & 24,7 & 12 & 14,1 & 8 & 9,4 & & 78 & 34 & 43,6 & 27 & 34,6 & 1 & 1,3 & 16 & 20,5 & \\
\hline \multicolumn{21}{|l|}{ Biberón } \\
\hline Sí & 11 & 7 & 63,6 & 2 & 18,2 & 2 & 18,2 & 0 & 0,0 & \multirow{2}{*}{0,557} & 6 & 5 & 83,3 & 0 & 0,0 & 0 & 0,0 & 1 & 16,7 & \multirow{2}{*}{0,161} \\
\hline No & 19 & 38 & 48,1 & 20 & 25,3 & 12 & 15,2 & 9 & 11,4 & & 81 & 31 & 28,3 & 30 & 37,0 & 1 & 1,2 & 19 & 23,5 & \\
\hline Chupón & & & & & & & & & & & & & & & & & & & & \\
\hline Sí & 0 & 0 & 0,0 & 0 & 0,0 & 0 & 0,0 & 0 & 0,0 & & 3 & 2 & 66,7 & 0 & 0,0 & 0 & 0,0 & 0 & 0,0 & 3 \\
\hline No & 90 & 45 & 50,0 & 22 & 24,4 & 14 & 15,6 & 9 & 10,0 & & 84 & 34 & 40,5 & 30 & 35,7 & 1 & 1,2 & 19 & 22,6 & \\
\hline Bruxismo & & & & & & & & & & & & & & & & & & & & \\
\hline Sí & 14 & 7 & 50,0 & 1 & 7,1 & 5 & 35,7 & 1 & 7,1 & $000^{\circ}$ & 10 & 3 & 30,0 & 5 & 50,0 & 0 & 0,0 & 2 & 20,0 & 0705 \\
\hline No & 76 & 38 & 50,0 & 21 & 27,6 & 9 & 11,8 & 8 & 10,5 & 0,090 & 77 & 33 & 42,9 & 25 & 32,5 & 1 & 1,3 & 18 & 23,4 & 0,225 \\
\hline Onicofag & & & & & & & & & & & & & & & & & & & & \\
\hline Sí & 7 & 3 & 42,9 & 4 & 57,1 & 0 & 0,0 & 0 & 0,0 & & 17 & 3 & 17,6 & 7 & 41,2 & 0 & 0,0 & 7 & 41,2 & \\
\hline No & 83 & 42 & 50,6 & 18 & 21,7 & 14 & 16,9 & 9 & 10,0 & 0,149 & 70 & 33 & 47,1 & 23 & 32,9 & 1 & 1,4 & 13 & 18,6 & 0,092 \\
\hline Deglució & atípica & & & & & & & & & & & & & & & & & & & \\
\hline Sí & 10 & 5 & 50,0 & 4 & 40,0 & 1 & 10,0 & 0 & 0,0 & 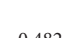 & 9 & 5 & 55,6 & 3 & 33,3 & 0 & 0,0 & 1 & 11,1 & 18 \\
\hline No & 80 & 40 & 50,0 & 18 & 22,5 & 13 & 16,3 & 9 & 11,3 & 0,702 & 78 & 31 & 39,7 & 27 & 34,6 & 1 & 1,3 & 19 & 24,4 & 0,448 \\
\hline Respiraci & n bucal & & & & & & & & & & & & & & & & & & & \\
\hline Sí & 20 & 7 & 35,0 & 5 & 25,0 & 3 & 15,0 & 5 & 25,0 & 007 & 11 & 5 & 45,5 & 4 & 36,4 & 0 & 0,0 & 2 & 18,2 & 5 \\
\hline No & 70 & 38 & 54,3 & 17 & 24,3 & 11 & 15,7 & 4 & 5,7 & $0,0 / 7$ & 76 & 31 & 40,8 & 26 & 34,2 & 1 & 1,3 & 18 & 23,7 & 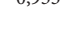 \\
\hline Objetos a & a boca & & & & & & & & & & & & & & & & & & & \\
\hline Sí & 9 & 5 & 55,6 & 1 & 11,1 & 3 & 33,3 & 0 & 0,0 & (2) & 3 & 1 & 33,3 & 2 & 66,7 & 0 & 0,0 & 0 & 0,0 & 50 \\
\hline No & 81 & 40 & 49,4 & 21 & 25,9 & 11 & 13,6 & 9 & 11,1 & 0,201 & 84 & 35 & 41,7 & 28 & 33,3 & 1 & 1,2 & 20 & 23,8 & 0,000 \\
\hline
\end{tabular}

Fuente: elaboración propia

Tabla 5. Relación entre tipo de hábito y presencia de maloclusión en el plano vertical

\begin{tabular}{|c|c|c|c|c|c|c|c|c|c|c|}
\hline \multirow{2}{*}{$\begin{array}{c}\text { Hábitos } \\
\text { bucales San } \\
\text { Luis Potosí }\end{array}$} & \multirow{2}{*}{$\begin{array}{l}\text { Tamaño de } \\
\text { la muestra }\end{array}$} & \multicolumn{2}{|c|}{ Mordida abierta } & \multirow{2}{*}{ P-valor } & \multicolumn{2}{|c|}{$\begin{array}{c}\text { Sobremordida } \\
\text { vertical }\end{array}$} & \multirow{2}{*}{ P-valor } & \multicolumn{2}{|c|}{$\begin{array}{l}\text { Mordida borde } \\
\text { a borde }\end{array}$} & \multirow{2}{*}{ P-valor } \\
\hline & & n & $\%$ & & $\mathbf{n}$ & $\%$ & & $\mathbf{n}$ & $\%$ & \\
\hline \multicolumn{11}{|l|}{ Succión digital } \\
\hline Sí & 36 & 4 & 11,1 & \multirow{2}{*}{0,112} & 9 & 25,0 & \multirow{2}{*}{0,355} & 3 & 8,3 & \multirow{2}{*}{0,542} \\
\hline No & 263 & 12 & 4,6 & & 55 & 20,9 & & 20 & 7,6 & \\
\hline \multicolumn{11}{|l|}{ Succión labial } \\
\hline Sí & 45 & 6 & 13,3 & \multirow{2}{*}{0,021} & 17 & 27,8 & \multirow{2}{*}{0,005} & 4 & 8,9 & \multirow{2}{*}{0,466} \\
\hline No & 254 & 10 & 3,9 & & 47 & 18,5 & & 19 & 7,5 & \\
\hline
\end{tabular}


(viene)

\begin{tabular}{|c|c|c|c|c|c|c|c|c|c|c|}
\hline \multirow{2}{*}{$\begin{array}{c}\text { Hábitos } \\
\text { bucales San } \\
\text { Luis Potosí }\end{array}$} & \multirow{2}{*}{$\begin{array}{l}\text { Tamaño de } \\
\text { la muestra }\end{array}$} & \multicolumn{2}{|c|}{ Mordida abierta } & \multirow[t]{2}{*}{ P-valor } & \multicolumn{2}{|c|}{$\begin{array}{c}\text { Sobremordida } \\
\text { vertical }\end{array}$} & \multirow[t]{2}{*}{ P-valor } & \multicolumn{2}{|c|}{$\begin{array}{c}\text { Mordida borde a } \\
\text { borde }\end{array}$} & \multirow[t]{2}{*}{ P-valor } \\
\hline & & n & $\%$ & & $\mathrm{n}$ & $\%$ & & $\mathrm{n}$ & $\%$ & \\
\hline \multicolumn{11}{|l|}{ Biberón } \\
\hline Sí & 12 & 1 & 8,3 & \multirow{2}{*}{0,490} & 2 & 16,7 & \multirow{2}{*}{0,507} & 0 & 0,0 & \multirow{2}{*}{0,376} \\
\hline No & 287 & 15 & 5,2 & & 62 & 21,6 & & 23 & 7,7 & \\
\hline \multicolumn{11}{|l|}{ Chupón } \\
\hline Sí & 0 & 0 & 0,0 & \multirow{2}{*}{ - } & 0 & 0,0 & & 0 & 0,0 & \multirow{2}{*}{ - } \\
\hline No & 299 & 16 & 5,4 & & 64 & 21,4 & & 27 & 9,0 & \\
\hline \multicolumn{11}{|l|}{ Bruxismo } \\
\hline Sí & 31 & 0 & 0,0 & \multirow{2}{*}{0,165} & 8 & 25,8 & \multirow{2}{*}{0,334} & 3 & 9,7 & \multirow{2}{*}{0,435} \\
\hline No & 268 & 16 & 6,0 & & 56 & 20,9 & & 20 & 7,5 & \\
\hline \multicolumn{11}{|l|}{ Onicofagia } \\
\hline Sí & 100 & 4 & 4,0 & \multirow[b]{2}{*}{0,330} & 17 & 17,0 & \multirow{2}{*}{0,121} & 7 & 7,0 & \multirow{2}{*}{0,474} \\
\hline No & 199 & 12 & 6,0 & & 47 & 23,6 & & 16 & 8,0 & \\
\hline Deglución atí & & & & & & & & & & \\
\hline Sí & 29 & 5 & 17,2 & ( & 5 & 17,2 & ( & 5 & 17,2 & \\
\hline No & 270 & 11 & 4,1 & 0,012 & 59 & 21,9 & 0,381 & 18 & 6,7 & 0,058 \\
\hline Respiración b & & & & & & & & & & \\
\hline Sí & 50 & 4 & 8,0 & & 9 & 18,0 & & 3 & 6,0 & \\
\hline No & 249 & 12 & 4,8 & 0,270 & 55 & 22,1 & 0,332 & 20 & 8,0 & 442 \\
\hline Objetos a la b & & & & & & & & & & \\
\hline Sí & 61 & 4 & 6,6 & & 14 & 23,0 & & 4 & 6,6 & \\
\hline No & 238 & 12 & 5,0 & 0,418 & 50 & 21,0 & 0,431 & 19 & 8,0 & 0,477 \\
\hline $\begin{array}{l}\text { Hábitos } \\
\text { bucales }\end{array}$ & Tamaño de & Mor & bierta & P-valor & Sob & dida & P-valor & Mor & orde & P-valor \\
\hline Medellín & la muestra & $\mathbf{n}$ & $\%$ & & $\mathbf{n}$ & $\%$ & & $\mathbf{n}$ & $\%$ & \\
\hline Succión digito & & & & & & & & & & \\
\hline Sí & 28 & 18 & 64,3 & & 3 & 10,7 & & 0 & 0,0 & \\
\hline No & 271 & 22 & 8,1 & 0,00 & 58 & 21,4 & 0,136 & 27 & 10,0 & 0,062 \\
\hline Succión labial & & & & & & & & & & \\
\hline Sí & 16 & 2 & 12,5 & 0636 & 5 & 31,3 & 0200 & 2 & 12,5 & 0424 \\
\hline No & 283 & 38 & 13,4 & 0,636 & 56 & 19,8 & 0,209 & 25 & 8,8 & 0,434 \\
\hline Biberón & & & & & & & & & & \\
\hline Sí & 7 & 1 & 14,3 & & 3 & 42,9 & 0153 & 0 & 0,0 & 0.512 \\
\hline No & 299 & 40 & 13,4 & 0,638 & 58 & 19,9 & 0,153 & 27 & 9,2 & 0,512 \\
\hline Chupón & & & & & & & & & & \\
\hline Sí & 6 & 2 & 33,3 & 0105 & 1 & 16,7 & 065 & 1 & 16,7 & 0426 \\
\hline No & 293 & 38 & 13,0 & 0,185 & 61 & 20,4 & 0,645 & 26 & 8,9 & 0,436 \\
\hline Bruxismo & & & & & & & & & & \\
\hline Sí & 35 & 1 & 2,9 & 0034 & 10 & 28,6 & 0146 & 4 & 11,4 & 201 \\
\hline No & 264 & 39 & 14,8 & 0,034 & 51 & 19,3 & 0,146 & 23 & 8,7 & 0,391 \\
\hline
\end{tabular}


(viene)

\begin{tabular}{|c|c|c|c|c|c|c|c|c|c|c|}
\hline \multirow{2}{*}{$\begin{array}{l}\text { Hábitos } \\
\text { bucales } \\
\text { Medellín }\end{array}$} & \multirow{2}{*}{$\begin{array}{l}\text { Tamaño de } \\
\text { la muestra }\end{array}$} & \multicolumn{2}{|c|}{ Mordida abierta } & \multirow[t]{2}{*}{ P-valor } & \multicolumn{2}{|c|}{$\begin{array}{l}\text { Sobremordida } \\
\text { vertical }\end{array}$} & \multirow[t]{2}{*}{ P-valor } & \multicolumn{2}{|c|}{$\begin{array}{l}\text { Mordida borde } \\
\text { a borde }\end{array}$} & \multirow[t]{2}{*}{ P-valor } \\
\hline & & $\mathrm{n}$ & $\%$ & & $\mathrm{n}$ & $\%$ & & $\mathbf{n}$ & $\%$ & \\
\hline \multicolumn{11}{|l|}{ Onicofagia } \\
\hline Sí & 56 & 5 & 8,9 & \multirow{2}{*}{0,195} & 13 & 23,2 & \multirow{2}{*}{0,339} & 6 & 10,7 & \multirow{2}{*}{0,393} \\
\hline No & 243 & 35 & 14,4 & & 48 & 19,8 & & 21 & 8,6 & \\
\hline \multicolumn{11}{|c|}{ Deglución atípica } \\
\hline Sí & 33 & 21 & 63,6 & \multirow{2}{*}{0,000} & 0 & 0,0 & \multirow[b]{2}{*}{0,000} & 3 & 9,1 & \multirow[b]{2}{*}{0,595} \\
\hline No & 266 & 19 & 7,1 & & 61 & 22,9 & & 24 & 9,0 & \\
\hline \multicolumn{11}{|c|}{ Respiración bucal } \\
\hline Sí & 61 & 7 & 11,5 & \multirow{2}{*}{0,402} & 12 & 19,7 & \multirow{2}{*}{0,516} & 6 & 9,8 & \multirow{2}{*}{0,485} \\
\hline No & 238 & 33 & 13,9 & & 49 & 20,6 & & 21 & 8,8 & \\
\hline \multicolumn{11}{|c|}{ Objetos a la boca } \\
\hline Sí & 3 & 0 & 0,0 & \multirow{2}{*}{0,649} & 1 & 33,3 & \multirow{2}{*}{0,497} & 0 & 0,0 & \multirow{2}{*}{0,752} \\
\hline No & 296 & 40 & 13,5 & & 60 & 20,3 & & 27 & 9,1 & \\
\hline
\end{tabular}

Fuente: elaboración propia

Tabla 6. Relación entre tipo de hábito y presencia de maloclusión en el plano horizontal

\begin{tabular}{|c|c|c|c|c|c|c|c|}
\hline \multirow{2}{*}{$\begin{array}{l}\text { Hábitos bucales } \\
\text { San Luis Potosí }\end{array}$} & \multirow{2}{*}{$\begin{array}{c}\text { Tamaño de la } \\
\text { muestra }\end{array}$} & \multicolumn{2}{|c|}{ Sobremordida horizontal } & \multirow{2}{*}{ P-valor } & \multicolumn{2}{|c|}{ Mordida cruzada anterior } & \multirow{2}{*}{ P-valor } \\
\hline & & $\mathbf{n}$ & $\%$ & & n & $\%$ & \\
\hline \multicolumn{8}{|l|}{ Succión digital } \\
\hline Sí & 36 & 5 & 13,9 & \multirow{2}{*}{0,418} & 0 & 0,0 & \multirow{2}{*}{0,121} \\
\hline No & 263 & 45 & 17,1 & & 16 & 6,1 & \\
\hline \multicolumn{8}{|l|}{ Succión labial } \\
\hline Sí & 45 & 11 & 24,4 & \multirow{2}{*}{0,102} & 2 & 4,4 & \multirow{2}{*}{0,557} \\
\hline No & 254 & 39 & 15,4 & & 14 & 5,5 & \\
\hline \multicolumn{8}{|l|}{ Biberón } \\
\hline Sí & 12 & 3 & 25,0 & \multirow{2}{*}{0,324} & 0 & 0,0 & \multirow{2}{*}{0,510} \\
\hline No & 287 & 47 & 16,4 & & 16 & 5,4 & \\
\hline \multicolumn{8}{|l|}{ Chupón } \\
\hline Sí & 0 & 0 & 0,0 & \multirow[b]{2}{*}{-} & 0 & 0,0 & \multirow[b]{2}{*}{-} \\
\hline No & 299 & 50 & 16,7 & & 28 & 9,4 & \\
\hline \multicolumn{8}{|l|}{ Bruxismo } \\
\hline Sí & 31 & 7 & 22,6 & \multirow{2}{*}{0,244} & 2 & 6,5 & \multirow{2}{*}{0,510} \\
\hline No & 268 & 43 & 16,0 & & 14 & 5,2 & \\
\hline \multicolumn{8}{|l|}{ Onicofagia } \\
\hline Sí & 100 & 20 & 20,0 & \multirow{2}{*}{0,180} & 3 & 3,0 & \multirow{2}{*}{0,157} \\
\hline No & 199 & 30 & 15,1 & & 13 & 6,5 & \\
\hline
\end{tabular}


(viene)

\begin{tabular}{|c|c|c|c|c|c|c|c|}
\hline \multirow{2}{*}{$\begin{array}{l}\text { Hábitos bucales } \\
\text { San Luis Potosí }\end{array}$} & \multirow{2}{*}{$\begin{array}{l}\text { Tamaño de la } \\
\text { muestra }\end{array}$} & \multicolumn{2}{|c|}{ Sobremordida horizontal } & \multirow{2}{*}{ P-valor } & \multicolumn{2}{|c|}{ Mordida cruzada anterior } & \multirow{2}{*}{ P-valor } \\
\hline & & $\mathbf{n}$ & $\%$ & & $\mathrm{n}$ & $\%$ & \\
\hline \multicolumn{8}{|l|}{ Deglución atípica } \\
\hline Sí & 29 & 4 & 13,8 & \multirow{2}{*}{0,446} & 3 & 10,3 & \multirow{2}{*}{0,194} \\
\hline No & 270 & 46 & 17,0 & & 13 & 4,8 & \\
\hline \multicolumn{8}{|l|}{ Respiración bucal } \\
\hline Sí & 50 & 6 & 12,0 & \multirow{2}{*}{0,224} & 7 & 14,0 & \multirow{2}{*}{0,008} \\
\hline No & 249 & 44 & 17,7 & & 9 & 3,6 & \\
\hline \multicolumn{8}{|l|}{ Objetos a la boca } \\
\hline Sí & 61 & 14 & 23,0 & \multirow{2}{*}{0,104} & 1 & 1,6 & \multirow{2}{*}{0,125} \\
\hline No & 238 & 36 & 15,1 & & 15 & 6,3 & \\
\hline \multirow{2}{*}{$\begin{array}{l}\text { Hábitos bucales } \\
\text { Medellín }\end{array}$} & \multirow{2}{*}{$\begin{array}{l}\text { Tamaño de la } \\
\text { muestra }\end{array}$} & \multicolumn{2}{|c|}{ Sobremordida horizontal } & \multirow{2}{*}{ P-valor } & \multicolumn{2}{|c|}{ Mordida cruzada anterior } & \multirow{2}{*}{ P-valor } \\
\hline & & $\mathbf{n}$ & $\%$ & & $\mathrm{n}$ & $\%$ & \\
\hline \multicolumn{8}{|l|}{ Succión digital } \\
\hline Sí & 28 & 8 & 28,6 & \multirow{2}{*}{0,009} & 2 & 7,1 & \multirow{2}{*}{0,498} \\
\hline No & 271 & 27 & 10,0 & & 26 & 9,6 & \\
\hline \multicolumn{8}{|l|}{ Succión labial } \\
\hline Sí & 16 & 2 & 12,5 & & 2 & 12,5 & \\
\hline No & 283 & 33 & 11,7 & 0,501 & 26 & 9,2 & 0,404 \\
\hline Biberón & & & & & & & \\
\hline Sí & 7 & 0 & 0,0 & 0414 & 1 & 14,3 & 0501 \\
\hline No & 299 & 35 & 12,0 & 0,414 & 27 & 9,2 & , \\
\hline Chupón & & & & & & & \\
\hline Sí & 6 & 1 & 16,7 & 0529 & 0 & 0,0 & 0.514 \\
\hline No & 293 & 34 & 11,6 & , & 28 & 9,6 & 0,017 \\
\hline Bruxismo & & & & & & & \\
\hline Sí & 35 & 1 & 2,9 & 0061 & 4 & 11,4 & 0420 \\
\hline No & 264 & 34 & 12,9 & 0,001 & 24 & 9,1 & $0,4<0$ \\
\hline Onicofagia & & & & & & & \\
\hline Sí & 56 & 7 & 12,5 & 0495 & 3 & 5,4 & 0190 \\
\hline No & 243 & 28 & 11,5 & S & 25 & 10,3 & סלוגים \\
\hline Deglución atípica & & & & & & & \\
\hline Sí & 33 & 4 & 12,1 & $0-50$ & 4 & 12,1 & $0=20$ \\
\hline No & 266 & 31 & 11,7 & 0,500 & 24 & 9,0 & 0,529 \\
\hline Respiración bucal & & & & & & & \\
\hline Sí & 61 & 10 & 16,4 & & 6 & 9,8 & \\
\hline No & 238 & 25 & 10,5 & 0,146 & 22 & 9,2 & 0,526 \\
\hline Objetos a la boca & & & & & & & \\
\hline Sí & 3 & 0 & 0,0 & 0687 & 0 & 0,0 & 0744 \\
\hline No & 296 & 35 & 11,8 & 0,687 & 28 & 9,5 & $0, / 44$ \\
\hline
\end{tabular}

Fuente: elaboración propia 
Tabla 7. Relación entre tipo de hábito y presencia de maloclusión en el plano transversal

\begin{tabular}{|c|c|c|c|c|}
\hline \multirow{2}{*}{$\begin{array}{l}\text { Hábitos bucales } \\
\text { San Luis Potosí }\end{array}$} & \multirow{2}{*}{$\begin{array}{c}\text { Tamaño } \\
\text { de la muestra }\end{array}$} & \multicolumn{2}{|c|}{ Mordida cruzada posterior } & \multirow{2}{*}{ P-valor } \\
\hline & & $\mathrm{n}$ & $\%$ & \\
\hline \multicolumn{5}{|l|}{ Succión digital } \\
\hline Sí & 36 & 5 & 13,9 & \multirow{2}{*}{0,031} \\
\hline No & 263 & 11 & 4,2 & \\
\hline \multicolumn{5}{|l|}{ Succión labial } \\
\hline Sí & 45 & 1 & 2,2 & \multirow{2}{*}{0,274} \\
\hline No & 254 & 15 & 5,9 & \\
\hline \multicolumn{5}{|l|}{ Biberón } \\
\hline Sí & 12 & 0 & 0,0 & \multirow{2}{*}{0,510} \\
\hline No & 287 & 16 & 5,6 & \\
\hline \multicolumn{5}{|l|}{ Chupón } \\
\hline Sí & 0 & 0 & 0,0 & \multirow[b]{2}{*}{-} \\
\hline No & 299 & 16 & 0,0 & \\
\hline \multicolumn{5}{|l|}{ Bruxismo } \\
\hline Sí & 31 & 2 & 6,5 & \multirow{2}{*}{0,510} \\
\hline No & 268 & 14 & 5,2 & \\
\hline \multicolumn{5}{|l|}{ Onicofagia } \\
\hline Sí & 100 & 8 & 8,0 & \multirow{2}{*}{0,122} \\
\hline No & 199 & 8 & 4,0 & \\
\hline \multicolumn{5}{|l|}{ Deglución atípica } \\
\hline Sí & 29 & 1 & 3,4 & \multirow{2}{*}{0,527} \\
\hline No & 270 & 15 & 5,6 & \\
\hline \multicolumn{5}{|l|}{ Respiración bucal } \\
\hline Sí & 50 & 2 & 4,0 & \multirow{2}{*}{0,481} \\
\hline No & 249 & 14 & 5,6 & \\
\hline \multicolumn{5}{|l|}{ Objetos a la boca } \\
\hline Sí & 61 & 2 & 3,3 & \multirow{2}{*}{0,331} \\
\hline No & 238 & 14 & 5,9 & \\
\hline \multirow{2}{*}{$\begin{array}{l}\text { Hábitos bucales } \\
\text { Medellín }\end{array}$} & \multirow{2}{*}{$\begin{array}{c}\text { Tamaño } \\
\text { de la muestra }\end{array}$} & \multicolumn{2}{|c|}{ Mordida cruzada posterior } & D-valor \\
\hline & & $\mathbf{n}$ & $\%$ & P-valor \\
\hline Succión digital & & & & \\
\hline Sí & 28 & 2 & 7,1 & 066 \\
\hline No & 271 & 20 & 7,4 & 0,60 \\
\hline Succión labial & & & & \\
\hline Sí & 16 & 1 & 6,3 & \\
\hline No & 283 & 21 & 7,4 & 0,667 \\
\hline Biberón & & & & \\
\hline Sí & 7 & 0 & 0,0 & 0582 \\
\hline No & 299 & 22 & 7,5 & 0,582 \\
\hline Chupón & & & & \\
\hline Sí & 6 & 0 & 0,0 & 063 \\
\hline No & 293 & 22 & 7,5 & 0,63 \\
\hline
\end{tabular}


(viene)

\begin{tabular}{|c|c|c|c|c|}
\hline \multirow{2}{*}{$\begin{array}{l}\text { Hábitos bucales } \\
\text { Medellín }\end{array}$} & \multirow{2}{*}{$\begin{array}{c}\text { Tamaño } \\
\text { de la muestra }\end{array}$} & \multicolumn{2}{|c|}{ Mordida cruzada posterior } & \multirow{2}{*}{ P-valor } \\
\hline & & $\mathrm{n}$ & $\%$ & \\
\hline \multicolumn{5}{|l|}{ Bruxismo } \\
\hline Sí & 35 & 1 & 2,9 & \multirow{2}{*}{0,242} \\
\hline No & 264 & 21 & 8,0 & \\
\hline \multicolumn{5}{|l|}{ Onicofagia } \\
\hline Sí & 56 & 2 & 3,6 & \multirow{2}{*}{0,181} \\
\hline No & 243 & 20 & 8,2 & \\
\hline \multicolumn{5}{|l|}{ Deglución atípica } \\
\hline Sí & 33 & 5 & 15,2 & \multirow{2}{*}{0,08} \\
\hline No & 266 & 17 & 6,4 & \\
\hline \multicolumn{5}{|l|}{ Respiración bucal } \\
\hline Sí & 61 & 6 & 9,8 & \multirow{2}{*}{0,278} \\
\hline No & 238 & 16 & 6,7 & \\
\hline \multicolumn{5}{|l|}{ Objetos a la boca } \\
\hline Sí & 3 & 0 & 0,0 & \multirow{2}{*}{0,794} \\
\hline No & 296 & 22 & 7,4 & \\
\hline
\end{tabular}

Fuente: elaboración propia

Como se observa en la Tabla 8, al comparar los resultados de la frecuencia de los diferentes hábitos con lo reportado en la literatura, se encontró que la onicofagia registrada por Garde et al. [10, p. 41] y Sadhan et al. [11] presenta menor frecuencia que la encontrada en nuestra investigación; por el contrario, Reyes Romagosa et al. [12] reportaron una mayor frecuencia a la nuestra.

Tabla 8. Comparación de prevalencia de hábitos bucales en la literatura

\begin{tabular}{|c|c|c|c|c|c|c|c|c|c|c|}
\hline Hábitos bucales & $\begin{array}{c}\text { Medellín } \\
\text { y San Luis } \\
\text { Potosí }\end{array}$ & $\begin{array}{c}\text { Karad, } \\
\text { India } \\
{[10]}\end{array}$ & $\begin{array}{c}\text { Ryyadh, } \\
\text { Arabia } \\
\text { Saudita } \\
\text { [11] }\end{array}$ & $\begin{array}{c}\text { Valencia, } \\
\text { España } \\
{[13]}\end{array}$ & $\begin{array}{c}\text { Domínguez } \\
{[15]}\end{array}$ & $\begin{array}{c}\text { Ryyadh, } \\
\text { Arabia } \\
\text { Saudita } \\
{[16]} \\
\end{array}$ & $\begin{array}{c}\text { Tirana, } \\
\text { Albania } \\
{[14]}\end{array}$ & $\begin{array}{c}\text { Manzanillo, } \\
\text { Cuba [12] }\end{array}$ & $\begin{array}{c}\text { Nigeria } \\
{[17]}\end{array}$ & $\begin{array}{c}\text { México } \\
\text { [17] }\end{array}$ \\
\hline Onicofagia & 26,1 & 5,8 & 15,6 & & & & & 42,8 & & 20,7 \\
\hline Respiración bucal & 18,6 & 4,3 & & 12 & & & 23,2 & & & 15,7 \\
\hline Bruxismo & 11,0 & 17,3 & & 2,36 & & & & & & \\
\hline Succión digital & 10,7 & 8,7 & 26,7 & 9,36 & 11,7 & 10,46 & 10,2 & 32,2 & & \\
\hline Succión labial & 10,2 & & & & & & & & 1,2 & \\
\hline Deglución atípica & 10,4 & 4,9 & & 22,82 & & & 16,2 & 27,2 & & \\
\hline Objetos a la boca & 10,7 & & & & & & & & & \\
\hline Biberón & 3,2 & 10,1 & & & & & & 133 & & \\
\hline Chupón & 1 & & & 0,73 & & 37,9 & 30 & J & & \\
\hline
\end{tabular}

Fuente: elaboración propia 
En relación con los hábitos de respiración bucal y bruxismo, encontramos que nuestros resultados son diferentes a los hallados por Paredes Gallardo et al. [13] y Garde et al. [10, p. 41]; sin embargo, en la respiración bucal se encontraron valores más similares a los reportados por Laganà et al. [14].

En la succión digital, con presencia en el 10,7\% del total de la muestra, se encontraron valores muy similares a los reportados por Domínguez et al. [15], Farsi y Salama [16], Paredes Gallardo et al. [13] y Garde et al. [10, p. 41]. En cuanto al hábito de succión labial, se encontraron valores muy superiores con respecto a lo hallado por Onyeaso [17].

Para la deglución atípica, se observan valores reportados con muy baja prevalencia como en Garde et al. [10, p. 40], con 4,9\%, y otros, mucho mayores, como Paredes Gallardo et al. [13, p. 261], Laganà et al. [14, p. 7] y Reyes Romagosa et al. [12, p. 608], con valores de 16,2 a $27,2 \%$. Estas grandes diferencias se hallaron igualmente en el hábito del uso de chupón. No se encontraron referencias reportadas para comparar el hábito de introducción de objetos en la boca.

Al comparar los estudios reportados en la literatura, es importante tener en cuenta las diferencias existentes en el tipo de muestra, como la edad o la ubicación geográfica, las cuales pueden tener algunos aspectos sociodemográficos e influir en los resultados presentados.

En las dos ciudades, los niños entre 10 y 12 años de edad presentaron mayor frecuencia de onicofagia, lo cual es consistente con lo expresado por Murrieta et al. [18] y Tanaka et al. [19], quienes refieren la onicofagia como el hábito oral más frecuente, probablemente debido al hecho de que este hábito puede ser transferencia de los hábitos de succión, que tienden a ser abandonados durante el tercer año de vida cuando comienza la onicofagia; mientras que el hábito de biberón tuvo mayor frecuencia en los pacientes entre 4 y 6 años en ambas ciudades.

En Medellín, se observó la respiración bucal con mayor frecuencia en los niños con edades entre 10 y 12 años, probablemente asociados a situaciones ambientales de la ciudad.

En cuanto a la relación molar permanente según la clasificación de Angle, tanto en San Luis Potosí como en Medellín se encontró una relación entre deglución atípica y clase III molar, lo cual no se encontró en ningún reporte de la literatura revisada.
En San Luis Potosí, los pacientes con hábitos de succión labial y deglución atípica presentaron una relación con presencia de mordida abierta anterior. Igualmente, se encontró una relación significativa entre la succión labial con mordida profunda. En Medellín, se observa una mordida abierta anterior en los pacientes que presentan succión digital y deglución atípica, dicha relación también fue encontrada por Jajoo et al. [20] y Urzal et al. [21], quienes relacionaron la mordida abierta sobre todo con el hábito de succión digital y el de empuje lingual.

$\mathrm{Al}$ analizar el vínculo entre la presencia de hábitos y el tipo de sobremordida horizontal, en San Luis Potosí se encontró una relación significativa entre los pacientes con hábito de respiración bucal y mordida cruzada anterior, sin hallar referencias similares en la literatura revisada; y en Medellín se encontró una relación entre quienes presentan succión digital con presencia de sobremordida horizontal, igual a lo reportado por Da Costa y Orenuga [22] y por Quashie-Williams et al. [23].

En San Luis Potosí, se encontró una relación en aquellos pacientes que presentan succión digital con mordida cruzada posterior, en contraste con lo reportado por Primožič et al. [24], quienes no encontraron que esta relación tuviera significancia estadística. Por el contrario, Da Costa y Orenunga [22, p. 40] reportan que encontraron este tipo de mordida en $8,65 \%$ de los pacientes con este hábito.

\section{Conclusiones}

El hábito de mayor prevalencia fue la onicofagia en el total de la muestra, seguido por la respiración bucal; y con valores similares encontramos: bruxismo, succión digital, succión labial, deglución atípica e introducción de objetos en la boca. Por último, solo un pequeño porcentaje usaba biberón y chupón.

La deglución atípica con mordida abierta presenta una relación estadísticamente significativa, por lo cual podemos inferir que este hábito incide en la presencia de dicha maloclusión; esto nos sugiere que la función deglutiva es un factor importante en el desarrollo del sistema estomatognático y puede ser un factor causal de esta maloclusión.

Aunque la onicofagia y la respiración bucal son de mayor prevalencia, en general no se debe descuidar el tratamiento apropiado de todos los hábitos en 
etapas tempranas de crecimiento y desarrollo en los niños, dado que pueden ser uno de los factores causales de maloclusiones futuras.

\section{Referencias}

[1] Clark J, Evans R. Funcional occlusion: I. A review. J Orthod. 2001;28(1):76-81. doi: http://dx.doi.org/ 10.1093/orto/28.1.76.

[2] Talley M, Katagiri M, Pérez HE. Casuística de maloclusiones clase I, clase II y clase III según Angle en el Departamento de Ortodoncia de la UnAM. Rev Odontol Mex. 2007;11(4):175-80.

[3] Espinal-Botero G, Muñoz A, Flores L, Ponce M, Nava J, González J. Frecuencia de maloclusión en las clínicas odontopediátricas de la Universidad de Antioquia, Colombia, y de la Universidad Autónoma de San Luis Potosí, México. Rev Nac Odontol. 2016:12(22):61-8. doi: http://dx.doi.org/10.16925/ od.v12i22.1206.

[4] Proffit WR, Fields HW, Sarver DM. Ortodoncia contemporánea. 4. ${ }^{\text {a }}$ ed. Barcelona: Elsevier; 2008.

[5] Tomita NE, Sheiham A, Bijella VT, Franco LJ. Relação entre determinantes socioeconômicos e hábitos bucais de risco para más-oclusões em pré-escolares. Pesqui Odontol Bras. 2000;14(2):169-75. doi: http:// dx.doi.org/10.1590/S1517-74912000000200013.

[6] Proffit WR, Fields H. Ortodoncia contemporánea. 3. ${ }^{\text {a }}$ ed. Barcelona: Mosby; 2001.

[7] Silva F, Alves Paz C. Epidemiology of malocclusion in children and adolescents: A critic review. Rev Gaúch Odontol. 2014;62(3):253-60. doi: http://dx. doi.org/10.1590/1981-8637201400030000041190.

[8] Leme M, Barbosa T, Castelo P, Gavião MB. Associations between psychological factors and the presence of deleterious oral habits in children and adolescents. J Clin Pediatr Dent. 2014;38(4):313-7.

[9] Agurto PV, Diaz RM, Cadiz OD, Bobenrieth FK. Oral bad habits frequency and its association with dentomaxilar abnormal development, in children three to six year old in Santiago Oriente. Rev Chil Pediatr. 1999;70(6):470-82.

[10] Garde JB, Suryavanshi RK, Jawale BA, Deshmukh V, Dadhe DP, Suryavanshi MK. An epidemiological study to know the prevalence of deleterious oral habits among 6 to 12 year old children. J Int Oral. 2014;6(1):39-43.

[11] Sadhan SA, Asma M. Zubair A. Oral habits, dental trauma, and oclusal characteristics among 4- to 12year-old institutionalized orphan children in Riyadh, Saudi Arabia. Spec Care Dentist. 2017;37(1):10-8. doi: http://dx.doi.org/10.1111/scd.2187.
[12] Reyes Romagosa D, Torres Pérez I, Quesada OL, Fernández M, Labrada Estrada H. Deforming oral habits in 5-11 years children. MEDISAN. 2014;18(5): 606-6012.

[13] Paredes Gallardo V, Paredes Cencillo C. Prevalencia de los hábitos bucales y alteraciones dentarias. An Pediatr. 2005;62(3):261-5. doi: http://dx.doi.org/ $101157 / 130718427$.

[14] Laganà G, Masucci C, Fabi F, Bollero P, Cozza P. Prevalence of malocclusions, oral habits and orthodontic treatment need in a 7 - to 15 -year-old school children population in Tirana. Prog Orth. 2013;14(1):1-7. doi: http://dx.doi.org/10.1186/21961042-14-12.

[15] Domínguez Reyes A, Galán González A, Aznar Martín T, Marín Castro I. Succión digital y parámetros oclusales: estudios en niños de 3 a 6 años de edad. Ortod Esp. 1999;39:143-7.

[16] Farsi NM, Salama FS. Sucking habits in Saudi children: Prevalence, contributing factors and effects on the primary dentition. Pediatr Dent. 1997;19(1): 28-33.

[17] Onyeaso CO. Oral habits among 7-10 year-old schoolchildren in Ibadan, Nigeria. East Afr Med J. 2004;81(1):16-21.

[18] Murrieta-Pruneda J, Martínez-Hernández L, Morales-Vázquez J, Linares-Vieyra C, González-Guevara $M$. Frecuencia y distribución de hábitos parafuncionales en un grupo de escolares mexicanos. Rev Nac Odontol. 2015;11(21):57-64. doi: http:// dx.doi.org/10.16925/od.v11i21.950.

[19] Tanaka OM, Vitral RWF, Tanaka GY, Guerrero AP, Camargo ES. Nailbiting, or onychophagia: A special habit. Am J Orthod Dentofacial Orthop. 2008;134(2):305-8. doi: http://dx.doi.org/10.1016/j. ajodo.2006.06.023.

[20] Jajoo S, Chunawala Y, Nadeem Bijle M, Shah R, Kamble A, Gaonkar N .Oral habits in school going children of Pune: A prevalence study. J Int Oral Health. 2015;7(10):96-101.

[21] Urzal V, Braga AC, Ferreira AP. Oral habits as risk factors for anterior open bite in the deciduous and mixed dentition - Cross-sectional study. Eur J Paediatr Dent. 2013;14(4):22-302.

[22] Da Costa OO, Orenuga OO. Dentofacial anomalies related to the digit sucking habit. Afr J Med Med Sci. 2002;31(3):239-42.

[23] Quashie-Williams R, Da Costa OO, Isiekwe MC. Oral habits, prevalence and effects on occlusion of 4-15 year old school children in Lagos, Nigeria. Niger Postgrad Med J. 2010;17(2):113-7. 
[24] PrimožičJ, FranchiL, Perinetti G, Richmond S, Ovsenik M. Influence of sucking habits and breathing pattern on palatal constriction in unilateral posterior cross bite - A controlled study. Eur J Orthod. 2013; (35):706-12. doi:http://dx.doi.org/10.1093/ejo/cjs086. 